\title{
Exendin-4, a glucagon-like peptide-1 receptor agonist, modulates hepatic fatty acid composition and $\Delta$-5-desaturase index in a murine model of non-alcoholic steatohepatitis
}

\author{
TAKUMI KAWAGUCHI ${ }^{1,2}$, MINORU ITOU ${ }^{1,3}$, EITARO TANIGUCHI $^{1}$ and MICHIO SATA ${ }^{1,2}$ \\ ${ }^{1}$ Division of Gastroenterology, Department of Medicine, ${ }^{2}$ Department of Disease Digestive Information and Research, \\ Kurume University School of Medicine; ${ }^{3}$ Kurume Clinical Pharmacology Clinic, \\ Medical Corporation Applied Bio-Pharmatech, Kurume 830-0011, Japan
}

Received December 20, 2013; Accepted May 28, 2014

DOI: 10.3892/ijmm.2014.1826

\begin{abstract}
Glucagon-like peptide-1 (GLP-1) is involved in the development of non-alcoholic steatohepatitis (NASH), which is characterized by fatty acid imbalance. The aim of this study was to investigate the effects of the GLP-1 receptor (GLP-1R) agonist, exendin-4 (Ex-4), on hepatic fatty acid metabolism and its key enzyme, $\Delta$-5-desaturase, in a murine model of NASH. NASH was induced in $\mathrm{db} / \mathrm{db}$ mice fed a methionine-choline deficient (MCD) diet. Ex-4 $(n=4)$ or saline [control $(\mathrm{CON}) ; \mathrm{n}=4$ ] was administered intraperitoneally for 8 weeks. Steatohepatitis activity was evaluated by non-alcoholic fatty liver disease (NAFLD) activity score. Hepatic fatty acid composition and $\Delta-5$-desaturase index were analyzed by gas chromatography. Ex-4 treatment significantly reduced body weight and the NAFLD activity score. Hepatic concentrations of long-chain saturated fatty acids (SFAs) were significantly higher in the Ex-4 group compared to the CON group $(23240 \pm 955$ vs. $31710 \pm 8436 \mu \mathrm{g} / \mathrm{g} \cdot \mathrm{liver}, \mathrm{P}<0.05)$. Ex-4 significantly reduced hepatic $n-3$ polyunsaturated fatty acid (PUFA)/n-6 PUFA ratio compared to the CON group $(13.83 \pm 3.15$ vs. $8.73 \pm 1.95, \mathrm{P}<0.05)$. In addition, the hepatic $\Delta$-5-desaturase index was significantly reduced in the Ex-4 group compared to the CON group $(31.1 \pm 12.4$ vs. $10.5 \pm 3.1$, $\mathrm{P}<0.05)$. In conclusion, the results showed that Ex-4 improved steatohepatitis in a murine model of NASH. Furthermore,
\end{abstract}

Correspondence to: Dr Takumi Kawaguchi, Division of Gastroenterology, Department of Medicine, Kurume University School of Medicine, 67 Asahi-machi, Kurume 830-0011, Japan

E-mail: takumi@med.kurume-u.ac.jp

Abbreviations: GLP-1, glucagon-like peptide-1; NASH, non-alcoholic steatohepatitis; Ex-4, exendin-4; GLP-1R, GLP-1 receptor; NAFLD, non-alcoholic fatty liver disease; PUFA, polyunsaturated fatty acid; $\mathrm{MCD}$, methionine-choline deficient; SFA, saturated fatty acid; MUFA, monounsaturated fatty acid

Key words: incretin, exenatide, steatosis, fatty acid composition, fatty acid desaturase
Ex-4 altered hepatic long-chain saturated and PUFA composition and reduced the $\Delta-5$-desaturase index. Thus, Ex-4 may improve NASH by regulating hepatic fatty acid metabolism.

\section{Introduction}

The incidence of non-alcoholic steatohepatitis (NASH) is rapidly increasing worldwide (1-4). NASH can be caused by various pathogenic mechanisms, including overeating, physical inactivity, diabetes mellitus, and medications $(5,6)$. The gut directly links to the liver through the portal vein and is involved in the development of NASH $(7,8)$. The gut secretes various hormones in the portal vein and regulates hepatic metabolism (9-11). Glucagon-like peptide-1 (GLP-1) is a gut hormone and is known to affect lipid metabolism in hepatocytes $(9,11)$.

Exendin-4 (Ex-4) is a long-acting GLP-1 receptor (GLP-1R) agonist. GLP-1R occurs in the pancreatic islets, kidney, lung, heart, stomach, intestine, thyroid gland, and numerous regions of the peripheral and central nervous system (12-14). GLP-1R also occurs in hepatocytes, and treatment with Ex-4 substantially reduces triglyceride stores in hepatoma cells (15). Similarly, GLP-1R agonist reduces steatosis severity in certain animal models of NASH (16-19). Findings of previous studies have also shown that reduced hepatic accumulation of triglycerides is mediated by GLP-1R agonist upregulation of hepatic 3-phosphoinositide-dependent kinase-1 activity, protein kinase $\mathrm{C} \zeta$ activity, peroxisome proliferator-activated receptor $\alpha$ activity, and fatty acid $\beta$-oxidation (15-19).

Fatty acids are an important triglyceride component. Fatty acids are a substrate of $\beta$-oxidation and yield large quantities of adenosine 5'-triphosphate (20). In addition, some polyunsaturated fatty acids (PUFAs) are a source of eicosanoids, which are biologically active substances. n-3 PUFAs are precursors of anti-inflammatory eicosanoids, including leukotriene B5, prostaglandin E3, and thromboxane B3 (21). On the other hand, n-6 PUFA are precursors of pro-inflammatory eicosanoids, including leukotriene B4, prostaglandin E2, and thromboxane B2 (21). A reduced n-3/n-6 PUFA ratio is a risk factor for chronic inflammatory diseases such as cardiovascular disease, inflammatory bowel disease, rheumatoid arthritis, and 
NASH (22-24). Thus, besides quantitative abnormality in fatty acids, qualitative abnormality in fatty acids is an important pathogenesis of NASH.

The production of pro- and anti-inflammatory eicosanoids is regulated by desaturases, which are rate-limiting enzymes of n-3 and n-6 PUFA cascades (25). $\Delta$-5-desaturase, also known as fatty acid desaturase 1 , removes two hydrogen atoms from dihomo $\gamma$-linolenic acid and synthesizes arachidonic acid. Upregulation of $\Delta-5$-desaturase activity promotes the production of pro-inflammatory eicosanoids (26). Notably, single-nucleotide polymorphisms in the $\Delta$-5-desaturase gene are associated with circulating high sensitivity $\mathrm{C}$-reactive protein levels in healthy young adults (27). Moreover, $\Delta$-5-desaturase activity is associated with aging (28), development of type 2 diabetes mellitus (29), and NASH (30). However, the effects of Ex-4 on hepatic fatty acid composition and $\Delta$-5-desaturase activity remain unclear.

The aim of this study was to investigate the effects of Ex-4 on severity of steatohepatitis, hepatic fatty acid composition, and $\Delta$-5-desaturase index in a murine model of NASH.

\section{Materials and methods}

Materials. Reagents were purchased from Wako Pure Chemical Industries, Ltd. (Osaka, Japan) unless otherwise indicated.

Animals. NASH was induced in $\mathrm{db} / \mathrm{db}$ mice fed a methionine-choline deficient (MCD) diet (31). Briefly, 5-week-old male $\mathrm{db} / \mathrm{db}$ mice (BKS.Cg- + Leprdb/+Leprdb/Jcl*) weighing 15-20 g were purchased from CLEA Japan, Inc. (Tokyo, Japan). The mice were housed individually in an air-conditioned room at $22 \pm 3^{\circ} \mathrm{C}$ and $55 \pm 10 \%$ humidity with a 12 -h light/dark cycle. The mice were fed a normal diet during a 1-week quarantine and acclimatization period, followed by the MCD diet (CLEA Japan, Inc.) and water ad libitum throughout the experimental period. All the rat experiments were conducted in accordance with the National Institutes of Health Guidelines for the Care and Use of Laboratory Animals and were approved by the University of Kurume Institutional Animal Care and Use Committee.

Treatment. Ex-4 (20 $\mu \mathrm{g} / \mathrm{kg}$; no. 24463, AnaSpec, Inc., Fremont, CA, USA) (Ex-4 group; $n=4)$ or saline [control (CON) group; $\mathrm{n}=4$ ] was administered intraperitoneally under anesthesia every morning for 8 weeks. At week 14, the mice were sacrificed by using ether anesthesia and the livers were obtained under anesthesia.

Measurement of body weight. Body weight was measured weekly, in the morning, through week 14 .

Liver histology. Random histological sampling was performed throughout this study as previously described (32,33). Liver samples were fixed overnight in $10 \%$ buffered formalin and embedded in paraffin. All sections were cut at a thickness of $5 \mu \mathrm{m}$ and stained with hematoxylin and eosin (H\&E) $(34,35)$.

Hepatic triglyceride content. Liver samples were fixed overnight in $10 \%$ buffered formalin. Sections were transferred to $70 \%$ ethanol and stained with Sudan IV (0.1\% Sudan IV dissolved in equal parts acetone and $70 \%$ ethanol) to evaluate triglyceride content (36).

Non-alcoholic fatty liver disease (NAFLD) activity. NAFLD activity was evaluated by the NAFLD activity score, in which the following findings were evaluated semi-quantitatively: steatosis ( $0-3$ points), lobular inflammation ( $0-2$ points), hepatocellular ballooning ( $0-2$ points), and fibrosis ( $0-4$ points) (37).

Fatty acid composition. Total liver fatty acids were extracted according to Folch et al (38). Fatty acid methyl esters were isolated and quantified by gas chromatography furnished with a flame-ionization detector. The fatty acids measured (and expressed as $\mu \mathrm{g} / \mathrm{g} \bullet$ liver) were: lauric, myristic, myristoleic, palmitic, palmitoleic, stearic, oleic, linoleic, $\gamma$-linolenic, linolenic, arachidic, eicosenoic, eicosadienoic, 5,8,11-eicosatrienoic, dihomo $\gamma$-linolenic, arachidonic, eicosapentaenoic, behenic, erucic, docosatetraenoic, docosapentaenoic, lignoceric, docosahexaenoic, and nervonic acid.

Classification of fatty acids. Fatty acids were classified as follows: saturated fatty acids (SFAs), the sum of all identified SAFs; atherogenic SFAs, the sum of lauric, myristic, and palmitic acids; thrombogenic SFAs, the sum of myristic, palmitic, and stearic acids; medium SFAs, the sum of SFAs containing 11-16 carbon atoms; long SFAs, the sum of SFAs containing $\geq 16$ carbon atoms; monounsaturated fatty acids (MUFAs), the sum of all identified MUFAs; PUFAs, the sum of all identified PUFAs; n-3 PUFAs, the sum of n-3 series PUFAs; n-6 PUFAs, the sum of n-6 series PUFA; $\Delta$-5-desatulase index, arachidonic acid $/ \gamma$-linolenic acid.

Statistical analysis. Data were expressed as mean \pm SD. Differences between two groups were analyzed by the Wilcoxon test (JMP version 10.0.2, SAS Institute, Inc., Cary, $\mathrm{NC}) . \mathrm{P} \leq 0.05$ was considered statistically significant.

\section{Results}

Effects of Ex-4 on body weight, appearance, and macroscopic appearance of the liver. In the CON group, body weight gradually increased to $\sim 50 \mathrm{~g}$ at week 14 (Fig. 1A). In the Ex-4 group, body weight gain stopped 1 week after the Ex-4 treatment and reached a plateau at $\sim 40 \mathrm{~g}$ at week 7 (Fig. 1A). Ex-4 significantly suppressed weight gain in MCD-fed $\mathrm{db} / \mathrm{db}$ mice.

Representative mice from the $\mathrm{CON}$ and Ex-4 groups are shown in Fig. 1B. The mouse from the Ex-4 group was smaller and had a good coat of fur in comparison to the mouse from the CON group (Fig. 1B).

A representative macroscopic image of the liver of $\mathrm{CON}$ and Ex-4 mice is shown in Fig. 1C. CON livers exhibited xanthochromia with swelling, while the Ex-4 livers were brown, with no swelling (Fig. 1C).

Effects of Ex-4 on hepatic histology, hepatic triglyceride content, and the NAFLD activity score. Representative images of hepatic histology and Sudan IV staining are shown in Fig. 2A. Steatosis, lobular inflammation, and hepatocyte ballooning were milder in the Ex-4 group compared to the CON group (Fig. 2A). Obvious hepatic fibrosis was not evident 


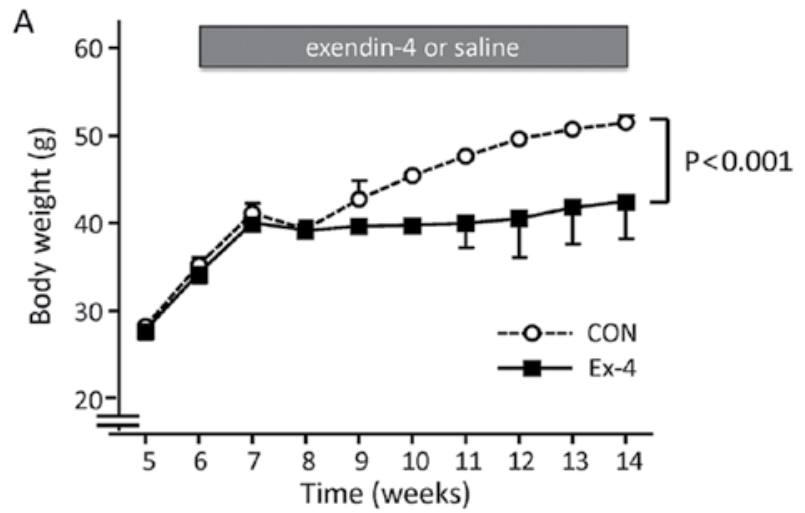

B

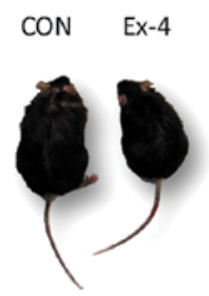

C

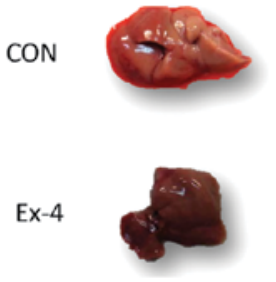

Figure 1. Effects of exendin-4 (Ex-4) on body weight, appearance, and macroscopic appearance of liver. (A) Changes in body weight. (B) Representative mice in the control (CON) and Ex-4 groups. (C) Representative livers from the $\mathrm{CON}$ and Ex-4 groups. $\mathrm{P} \leq 0.05$ was considered statistically significant.

A

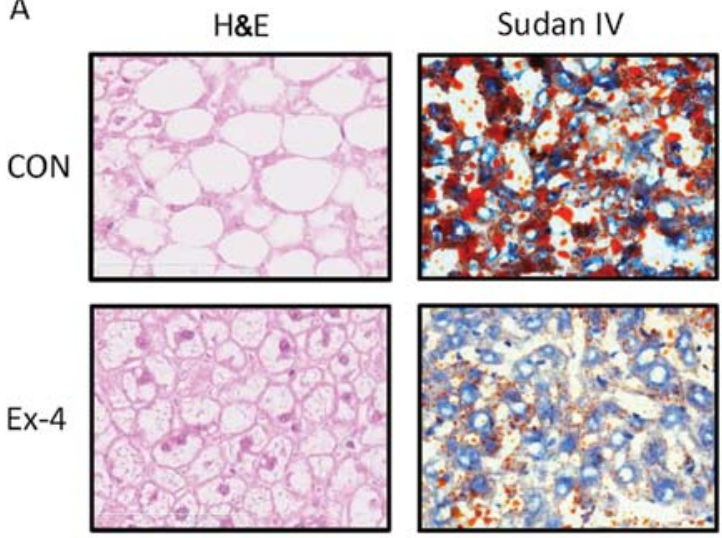

B

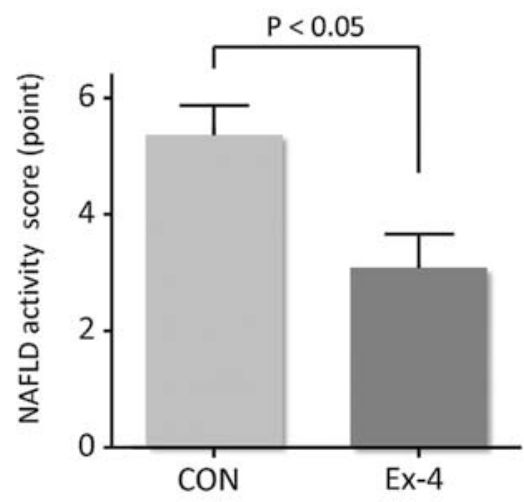

Figure 2. Effects of exendin-4 (Ex-4) on hepatic histology, hepatic triglyceride content, and non-alcoholic fatty liver disease (NAFLD) activity score. (A) Representative images of hepatic histology and Sudan IV staining. (B) The difference in the NAFLD activity score between the Ex-4 and control (CON) groups. $\mathrm{P} \leq 0.05$ was considered statistically significant. Hematoxylin and eosin staining (H\&E).
Table I. Effects of Ex-4 on hepatic SFA.

\begin{tabular}{lllcc}
\hline SFA type & Unit & CON & Ex-4 & P \\
\hline SFA & $\mu \mathrm{g} / \mathrm{g} \cdot l i v e r$ & $17838 \pm 3248$ & $27541 \pm 9273$ & N.S. \\
Atherogenic & $\mu \mathrm{g} / \mathrm{g} \cdot l i v e r$ & $13414 \pm 2981$ & $22457 \pm 8670$ & N.S. \\
Thrombogenic & $\mu \mathrm{g} / \mathrm{g} \cdot l i v e r$ & $17605 \pm 3244$ & $27210 \pm 9260$ & N.S. \\
Medium-chain & $\mu \mathrm{g} / \mathrm{g} \cdot$ liver & $15233 \pm 3554$ & $25186 \pm 9799$ & N.S. \\
Long-chain & $\mu \mathrm{g} / \mathrm{g} \cdot$ liver & $23240 \pm 955$ & $31710 \pm 8436$ & $<0.05$
\end{tabular}

Ex-4, exendin-4; SFA, saturated fatty acid; CON, control; N.S., not significant.

Table II. Effects of Ex-4 on hepatic MUFAs and PUFAs.

\begin{tabular}{lcccc}
\hline Acid type & Unit & CON & Ex-4 & P \\
\hline MUFA & $\mu \mathrm{g} / \mathrm{g} \cdot$ liver & $20355 \pm 6701$ & $34965 \pm 14485$ & N.S. \\
PUFA & $\mu \mathrm{g} / \mathrm{g} \cdot$ liver & $18410 \pm 791$ & $25986 \pm 8050$ & $<0.05$ \\
n-3 PUFA & $\mu \mathrm{g} / \mathrm{g} \cdot$ liver & $2218.5 \pm 415.8$ & $1992.4 \pm 288.7$ & N.S. \\
n-6 PUFA & $\mu \mathrm{g} / \mathrm{g} \cdot$ liver & $16166 \pm 943$ & $23937 \pm 7845$ & $<0.05$ \\
n-3 PUFA/ & Ratio & $13.83 \pm 3.15$ & $8.73 \pm 1.95$ & $<0.05$ \\
n-6 PUFA & & & & \\
\hline
\end{tabular}

Ex-4, exendin-4; MUFA, monounsaturated fatty acid; PUFA, polyunsaturated fatty acid; CON, control; N.S., not significant.

in either group. Hepatic triglyceride content was depleted in the Ex-4 group in comparison to the CON group (Fig. 2A).

The NAFLD activity score was significantly lower in the Ex-4 group than in the CON group (Fig. 2B).

Effects of Ex-4 on hepatic SFA. There was no significant difference in the hepatic SFA content of the CON and Ex-4 groups (Table I). No significant difference between the groups was observed in the hepatic content of atherogenic, thrombogenic, and medium-chain SFA. However, long-chain SFA content was significantly higher in the Ex-4 group compared to the CON group (Table I).

We also examined the hepatic content of each long-chain SFA component and found no significant differences in the palmitic, stearic, behenic, and lignoceric acid. However, hepatic arachidic acid was significantly higher in the Ex-4 group compared to the CON group (Fig. 3A-E).

Effects of Ex-4 on hepatic MUFAs and PUFAs. Hepatic MUFA content did not significantly differ between groups (Table II). However, hepatic PUFA content was significantly higher in the Ex-4 group compared to the CON group. Similarly, hepatic n-6 PUFA content and the n-3 PUFA/n-6 PUFA ratio were significantly higher in the Ex-4 group compared to the CON group (Table II).

We also assessed the hepatic content of each n-6 PUFA component and found no significant difference in arachidonic acid. However, the hepatic content of linoleic acid, $\gamma$-linolenic acid, and dihomo $\gamma$-linolenic acid was significantly higher in the Ex-4 group compared to the CON group (Fig. 4A-D). By contrast, hepatic $\Delta-5$-desaturase index in the Ex-4 group 

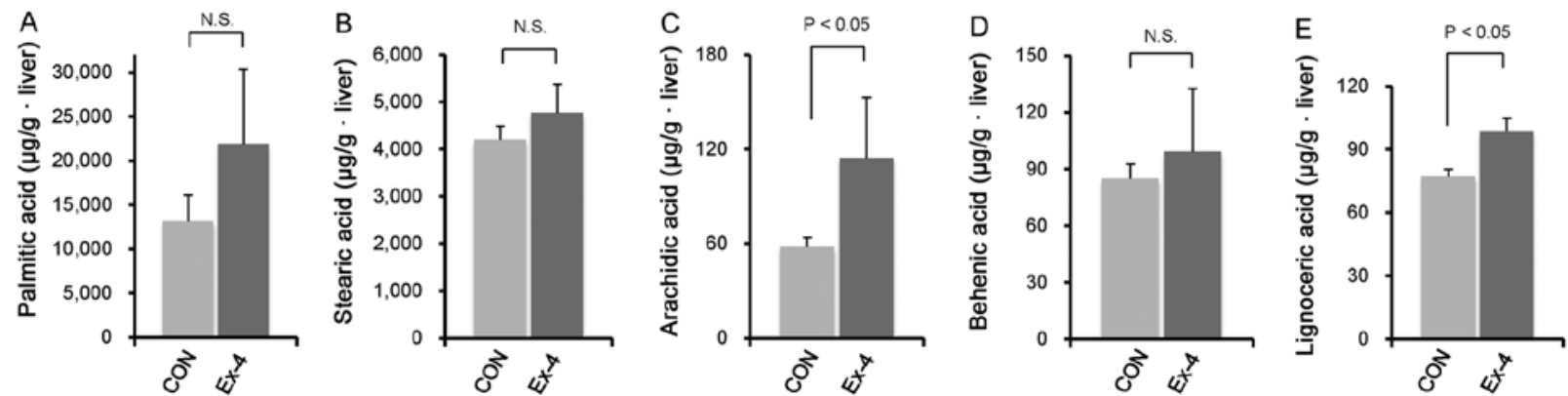

Figure 3. Effects of exendin-4 (Ex-4) on hepatic long-chain saturated fatty acids (SFAs). (A) Palmitic acid, (B) stearic acid, (C) arachidic acid, (D) behenic acid, and (E) lignoceric acid. $\mathrm{P} \leq 0.05$ was considered statistically significant. Control (CON), not significant (N.S.).
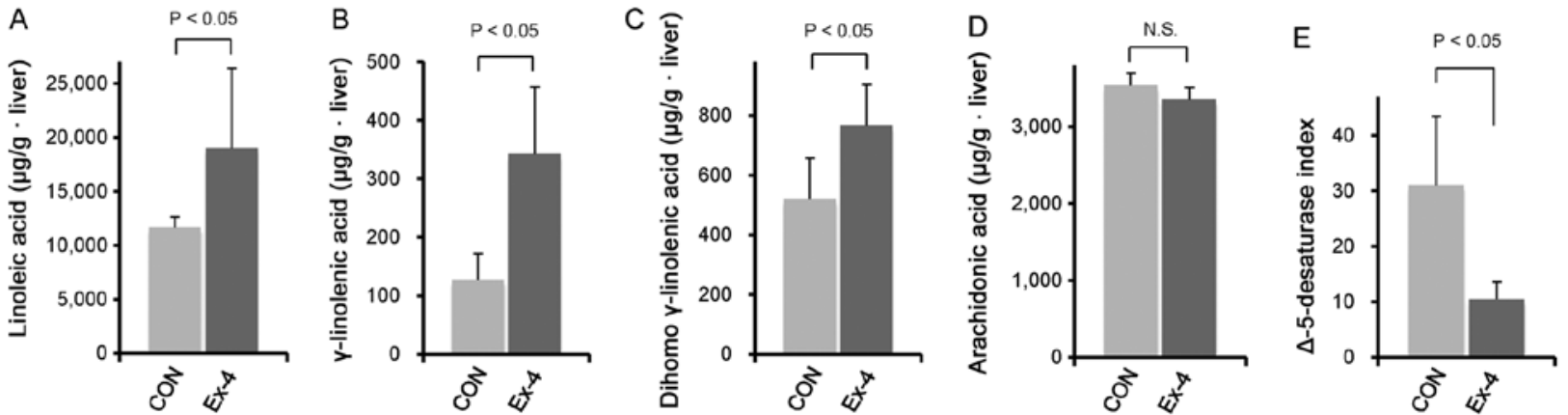

Figure 4. Effects of exendin-4 (Ex-4) on hepatic n-6 polyunsaturated fatty acids (PUFAs). (A) Linoleic acid, (B) $\gamma$-linolenic acid, (C) dihomo $\gamma$-linolenic acid, (D) arachidonic acid, and (E) $\Delta$-5-desaturase index. P $\leq 0.05$ was considered statistically significant. Control (CON), not significant (N.S.).

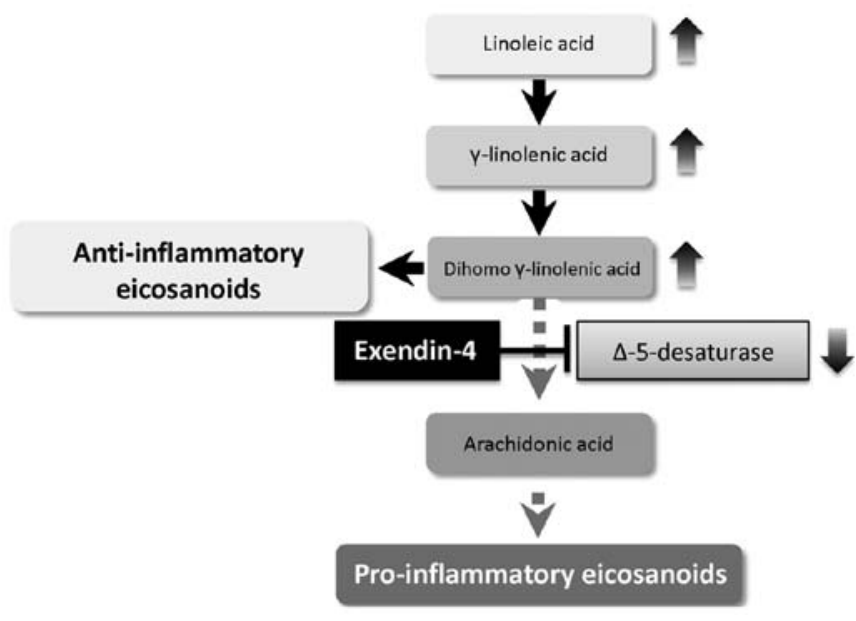

Figure 5. A scheme for exendin-4 (Ex-4)-caused alterations in lipid metabolism. Ex-4 may inhibit $\Delta$-5-desaturase activity, resulting in arachidonic acid production and subsequent pro-inflammatory eicosanoids. Inhibition of $\Delta$-5-desaturase activity also increases the hepatic content of dihomo $\gamma$-linolenic acid and subsequent anti-inflammatory eicosanoids.

was approximately one-third of that in the CON group. Ex-4 treatment significantly reduced hepatic $\Delta$-5-desaturase index compared to the CON group (Fig. 4E).

\section{Discussion}

Results of this study have shown that Ex-4 inhibited body weight gain and improved NASH in MCD diet-fed $\mathrm{db} / \mathrm{db}$ mice. Ex-4 also altered hepatic fatty acid composition with a decrease in $\Delta$-5-desaturase index. Thus, Ex-4 may improve NASH by altering the hepatic fatty acid composition in a murine model of NASH.

The effects of the GLP-1R agonist Ex-4 on NASH were examined. The results showed that Ex-4 significantly suppressed body weight gain and the NAFLD activity score in MCD diet-fed db/db mice. GLP-1R expression is downregulated in a NASH rat model as well as in patients with NASH (16). Moreover, GLP-1R agonist improves NASH in various animal models, including high-fat diet-fed rats (16), ob/ob mice (17,18), and diabetic male ApoE(-/-) mice (19). The GLP-1R agonist also reduced body weight and the NAFLD activity score in patients with NASH (39). Thus, our results are consistent with previous reports in this regard. Possible mechanisms for GLP-1R agonist-induced NASH improvement include the upregulation of insulin sensitivity, peroxisome proliferator-activated receptor $\alpha$ activity, and fatty acid $\beta$-oxidation $(15,16,40,41)$. However, the effects of GLP-1R agonist in hepatic fatty acid composition remain unclear.

In general, long-chain SFAs promote inflammation and progression of NAFLD $(42,43)$. However, results of this study have shown that Ex-4 significantly increased the hepatic content of long-chain SFAs, in particular the arachidic and lignoceric acids. Although the reason for the discrepancy between previous reports and our findings remains unclear, certain SFAs, including arachidic and lignoceric acids are not correlated with insulin resistance, a feature of NASH (44). Furthermore, arachidic acid improves lipid metabolism by enhancing apoB secretion (45). Lignoceric acid is a precursor of ceramide, thus an increase in hepatic lignoceric 
acid content indicates a decrease in ceramide synthesis. Recently, Kurek et al showed that inhibition of ceramide synthesis reduces hepatic lipid accumulation in a rat model of NAFLD (46). This finding suggests that Ex-4 improves lipid metabolism through alterations in arachidic and lignoceric acids in a murine model of NASH.

Although hepatic MUFA content was not altered by Ex-4 treatment, hepatic PUFA content was increased. Ex-4 increased the hepatic content of $\mathrm{n}-6$ PUFAs such as linoleic acid, $\gamma$-linolenic acid, and dihomo $\gamma$-linolenic acid. These $n-6$ PUFAs are precursors of pro-inflammatory eicosanoids and are involved in the development of NASH $(22,47)$. Thus, our findings are different from those of previous studies. However, a possible explanation for the discrepancy is an Ex-4-induced alteration in n-6 PUFA metabolism. $\Delta$-5-desaturase is a rate-limiting enzyme of $n-6$ PUFA metabolism that increases the production of pro-inflammatory eicosanoids (48). An oligonucleotide microarray analysis using human liver tissue showed that $\Delta-5$-desaturase is upregulated in patients with NASH (30). In this study, we have found that the $\Delta$-5-desaturase index was significantly reduced by Ex- 4 treatment, indicating that Ex-4 inhibits $\Delta-5$-desaturase activity and subsequently suppresses the production of pro-inflammatory eicosanoids (Fig. 5). In addition, the inhibition of $\Delta$-5-desaturase activity increases hepatic contents of dihomo $\gamma$-linolenic acid, which is a precursor of anti-inflammatory eicosanoids (Fig. 5). López-Vicario et al recently showed that a $\Delta$-5-desaturase inhibitor, CP-24879, significantly reduces intracellular lipid accumulation and inflammatory injury in hepatocytes in vitro (30), supporting our hypothesis. Thus, our findings together with those of previous studies suggest that suppression of $\Delta-5$-desaturase activity could be a new therapeutic strategy for NASH.

In conclusion, the results of the present study have shown that Ex-4 suppressed body weight gain and improved steatohepatitis in a murine model of NASH. Ex-4 also altered hepatic fatty acid composition with a decrease in $\Delta$-5-desaturase index. These findings suggest that Ex-4 improves NASH by modulating hepatic fatty acid metabolism.

\section{Acknowledgements}

This study was supported, in part, by Health and Labour Sciences Research Grants for Research on Hepatitis from the Ministry of Health, Labour and Welfare of Japan.

\section{References}

1. Williams CD, Stengel J, Asike MI, et al: Prevalence of nonalcoholic fatty liver disease and nonalcoholic steatohepatitis among a largely middle-aged population utilizing ultrasound and liver biopsy: a prospective study. Gastroenterology 140 : 124-131, 2011.

2. Ono M and Saibara T: Clinical features of nonalcoholic steatohepatitis in Japan: Evidence from the literature. J Gastroenterol 41 : 725-732, 2006

3. Sumida Y, Yoneda M, Hyogo H, et al: A simple clinical scoring system using ferritin, fasting insulin, and type IV collagen $7 \mathrm{~S}$ for predicting steatohepatitis in nonalcoholic fatty liver disease. J Gastroenterol 46: 257-268, 2011.

4. Eguchi Y, Hyogo H, Ono M, et al: Prevalence and associated metabolic factors of nonalcoholic fatty liver disease in the general population from 2009 to 2010 in Japan: a multicenter large retrospective study. J Gastroenterol 47: 586-595, 2012.
5. Nakahara T, Hyogo H, Yoneda M, et al: Type 2 diabetes mellitus is associated with the fibrosis severity in patients with nonalcoholic fatty liver disease in a large retrospective cohort of Japanese patients. J Gastroenterol: Nov 26, 2013 (Epub ahead of print).

6. Angulo P: Nonalcoholic fatty liver disease. N Engl J Med 346: 1221-1231, 2002.

7. Imajo K, Fujita K, Yoneda M, et al: Hyperresponsivity to low-dose endotoxin during progression to nonalcoholic steatohepatitis is regulated by leptin-mediated signaling. Cell Metab 16: 44-54, 2012.

8. Smith K: Microbiota: Gut microbiota produce alcohol in patients with NASH. Nat Rev Gastroenterol Hepatol 9: 687, 2012.

9. Mells JE and Anania FA: The role of gastrointestinal hormones in hepatic lipid metabolism. Semin Liver Dis 33: 343-357, 2013.

10. Itou M, Kawaguchi T, Taniguchi E, et al: Altered expression of glucagon-like peptide-1 and dipeptidyl peptidase IV in patients with $\mathrm{HCV}$-related glucose intolerance. J Gastroenterol Hepatol 23: 244-251, 2008

11. Itou M, Kawaguchi T, Taniguchi E and Sata M: Dipeptidyl peptidase-4: a key player in chronic liver disease. World J Gastroenterol 19: 2298-2306, 2013.

12. Drucker DJ: The biology of incretin hormones. Cell Metab 3: 153-165, 2006.

13. Gier B, Butler PC, Lai CK, Kirakossian D, DeNicola MM and Yeh MW: Glucagon like peptide-1 receptor expression in the human thyroid gland. J Clin Endocrinol Metab 97: 121-131, 2012.

14. Broide E, Bloch O, Ben-Yehudah G, Cantrell D, Shirin H and Rapoport MJ: GLP-1 receptor is expressed in human stomach mucosa: analysis of its cellular association and distribution within gastric glands. J Histochem Cytochem 61: 649-658, 2013.

15. Gupta NA, Mells J, Dunham RM, et al: Glucagon-like peptide-1 receptor is present on human hepatocytes and has a direct role in decreasing hepatic steatosis in vitro by modulating elements of the insulin signaling pathway. Hepatology 51: 1584-1592, 2010.

16. Svegliati-Baroni G, Saccomanno S, Rychlicki C, et al: Glucagon-like peptide-1 receptor activation stimulates hepatic lipid oxidation and restores hepatic signalling alteration induced by a high-fat diet in nonalcoholic steatohepatitis. Liver Int 31: 1285-1297, 2011

17. Trevaskis JL, Griffin PS, Wittmer C, et al: Glucagon-like peptide-1 receptor agonism improves metabolic, biochemical, and histopathological indices of nonalcoholic steatohepatitis in mice. Am J Physiol Gastrointest Liver Physiol 302: G762-G772, 2012.

18. Dhanesha N, Joharapurkar A, Shah G, et al: Treatment with exendin-4 improves the antidiabetic efficacy and reverses hepatic steatosis in glucokinase activator treated $\mathrm{db} / \mathrm{db}$ mice. Eur J Pharmacol 714: 188-192, 2013.

19. Panjwani N, Mulvihill EE, Longuet C, et al: GLP-1 receptor activation indirectly reduces hepatic lipid accumulation but does not attenuate development of atherosclerosis in diabetic male ApoE(-/-) mice. Endocrinology 154: 127-139, 2013.

20. Stumpf PK: Metabolism of fatty acids. Annu Rev Biochem 38: 159-212, 1969.

21. Calder PC: N-3 polyunsaturated fatty acids and inflammation: from molecular biology to the clinic. Lipids 38: 343-352, 2003.

22. Patterson E, Wall R, Fitzgerald GF, Ross RP and Stanton C: Health implications of high dietary omega- 6 polyunsaturated Fatty acids (Review). J Nutr Metab 2012: e539426, 2012.

23. Puri P, Baillie RA, Wiest MM, et al: A lipidomic analysis of nonalcoholic fatty liver disease. Hepatology 46: 1081-1090, 2007.

24. Puri P, Wiest MM, Cheung O, et al: The plasma lipidomic signature of nonalcoholic steatohepatitis. Hepatology 50: 1827-1838, 2009.

25. Poudel-Tandukar K, Sato M, Ejima Y, et al: Relationship of serum fatty acid composition and desaturase activity to C-reactive protein in Japanese men and women. Atherosclerosis 220: 520-524, 2012 .

26. Chuang LT, Thurmond JM, Liu JW, Mukerji P, Bray TM and Huang YS: Effect of conjugated linoleic acid on Delta-5 desaturase activity in yeast transformed with fungal Delta-5 desaturase gene. Mol Cell Biochem 265: 11-18, 2004.

27. Roke K, Ralston JC, Abdelmagid S, et al: Variation in the FADS1/2 gene cluster alters plasma n-6 PUFA and is weakly associated with hsCRP levels in healthy young adults. Prostaglandins Leukot Essent Fatty Acids 89: 257-263, 2013.

28. Maniongui C, Blond JP, Ulmann L, Durand G, Poisson JP and Bézard J: Age-related changes in delta 6 and delta 5 desaturase activities in rat liver microsomes. Lipids 28: 291-297, 1993. 
29. Kröger J and Schulze MB: Recent insights into the relation of $\Delta 5$ desaturase and $\Delta 6$ desaturase activity to the development of type 2 diabetes. Curr Opin Lipidol 23: 4-10, 2012.

30. López-Vicario C, González-Périz A, Rius B, et al: Molecular interplay between $\Delta 5 / \Delta 6$ desaturases and long-chain fatty acids in the pathogenesis of non-alcoholic steatohepatitis. Gut 63: 344-355, 2014.

31. Yamaguchi K, Yang L, McCall S, et al: Inhibiting triglyceride synthesis improves hepatic steatosis but exacerbates liver damage and fibrosis in obese mice with nonalcoholic steatohepatitis Hepatology 45: 1366-1374, 2007.

32. Kawaguchi T, Sakisaka S, Sata M, Mori M and Tanikawa K: Different lobular distributions of altered hepatocyte tight junctions in rat models of intrahepatic and extrahepatic cholestasis. Hepatology 29: 205-216, 1999.

33. Kawaguchi T, Sakisaka S, Mitsuyama K, et al: Cholestasis with altered structure and function of hepatocyte tight junction and decreased expression of canalicular multispecific organic anion transporter in a rat model of colitis. Hepatology 31 : $1285-1295,2000$.

34. Kawaguchi T, Yoshida T, Harada M, et al: Hepatitis $\mathrm{C}$ virus down-regulates insulin receptor substrates 1 and 2 through up-regulation of suppressor of cytokine signaling 3. Am J Pathol 165: 1499-1508, 2004

35. Kawaguchi T,Ide T, TaniguchiE, et al: Clearance of HCV improves insulin resistance, beta-cell function, and hepatic expression of insulin receptor substrate 1 and 2 . Am J Gastroenterol 102: 570-576, 2007.

36. Krishna SM, Seto SW, Moxon JV, et al: Fenofibrate increases high-density lipoprotein and sphingosine 1 phosphate concentrations limiting abdominal aortic aneurysm progression in a mouse model. Am J Pathol 181: 706-718, 2012.

37. Kleiner DE, Brunt EM, Van Natta M, et al: Design and validation of a histological scoring system for nonalcoholic fatty liver disease. Hepatology 41: 1313-1321, 2005.

38. Folch J, Lees M and Sloane Stanley GH: A simple method for the isolation and purification of total lipides from animal tissues. J Biol Chem 226: 497-509, 1957.
39. Kenny PR, Brady DE, Torres DM, Ragozzino L, Chalasani N and Harrison SA: Exenatide in the treatment of diabetic patients with non-alcoholic steatohepatitis: a case series. Am J Gastroenterol 105: 2707-2709, 2010.

40. Ding X, Saxena NK, Lin S, Gupta NA and Anania FA: Exendin-4, a glucagon-like protein-1 (GLP-1) receptor agonist, reverses hepatic steatosis in ob/ob mice. Hepatology 43: 173-181, 2006.

41. Gupta NA, Kolachala VL, Jiang R, et al: The glucagon-like peptide-1 receptor agonist Exendin 4 has a protective role in ischemic injury of lean and steatotic liver by inhibiting cell death and stimulating lipolysis. Am J Pathol 181: 1693-1701, 2012.

42. Leamy AK, Egnatchik RA and Young JD: Molecular mechanisms and the role of saturated fatty acids in the progression of non-alcoholic fatty liver disease. Prog Lipid Res 52: 165-174, 2013.

43. Matsumori R, Miyazaki T, Shimada K, et al: High levels of very long-chain saturated fatty acid in erythrocytes correlates with atherogenic lipoprotein profiles in subjects with metabolic syndrome. Diabetes Res Clin Pract 99: 12-18, 2013.

44. Kusunoki M, Tsutsumi K, Nakayama M, et al: Relationship between serum concentrations of saturated fatty acids and unsaturated fatty acids and the homeostasis model insulin resistance index in Japanese patients with type 2 diabetes mellitus. J Med Invest 54: 243-247, 2007.

45. Arrol S, Mackness MI and Durrington PN: The effects of fatty acids on apolipoprotein B secretion by human hepatoma cells (HEP G2). Atherosclerosis 150: 255-264, 2000.

46. Kurek K, Piotrowska DM, Wiesiolek-Kurek P, et al: Inhibition of ceramide de novo synthesis reduces liver lipid accumulation in rats with nonalcoholic fatty liver disease. Liver Int: Sep 25, 2013 (Epub ahead of print).

47. Araya J, Rodrigo R, Videla LA, et al: Increase in long-chain polyunsaturated fatty acid $n-6 / n-3$ ratio in relation to hepatic steatosis in patients with non-alcoholic fatty liver disease. Clin Sci (Lond) 106: 635-643, 2004.

48. de Gomez Dumm IN, de Alaniz MJ and Brenner RR: Effect of dietary fatty acids on delta 5 desaturase activity and biosynthesis of arachidonic acid in rat liver microsomes. Lipids 18: 781-788, 1983. 